\title{
Positivity for the clamped plate equation under high tension
}

\author{
Sascha Eichmann ${ }^{1}$ (D) Reiner M. Schätzle ${ }^{1}$
}

Received: 22 June 2021 / Accepted: 7 January 2022 / Published online: 2 February 2022

(c) The Author(s) 2022

\begin{abstract}
In this article, we consider positivity issues for the clamped plate equation with high tension $\gamma>0$. This equation is given by $\Delta^{2} u-\gamma \Delta u=f$ under clamped boundary conditions. Here, we show that given a positive $f$, i.e. upwards pushing, we find a $\gamma_{0}>0$ such that for all $\gamma \geq \gamma_{0}$ the bending $u$ is indeed positive. This $\gamma_{0}$ only depends on the domain and the ratio of the $L^{1}$ and $L^{\infty}$ norm of $f$. In contrast to a recent result by Cassani and Tarsia, our approach is valid in all dimensions.
\end{abstract}

Keywords Bi-Laplace equation $\cdot$ Maximum principle $\cdot$ High tension

Mathematics Subject Classification 35B09 $\cdot 35 \mathrm{~B} 30 \cdot 35 \mathrm{~B} 50 \cdot 35 \mathrm{~J} 40$

\section{Introduction}

The Boggio-Hadamard conjecture states, that for a given convex, open, bounded set $\Omega \subseteq \mathbb{R}^{n}$, an $f \geq 0$ and outer normal $\nu$ of $\Omega$, a solution $u$ to

$$
\left\{\begin{array}{cc}
\Delta^{2} u=f & \text { in } \Omega \\
u=\partial_{\nu} u=0 & \text { on } \partial \Omega .
\end{array}\right.
$$

is nonnegative, i.e. $u \geq 0$ (cf. [26, 27]). Problem (1.1) models the bending $u$ of a clamped plate $\Omega$ under a force $f$. Hence the problem can be restated as:

Does upward pushing yield upward bending?

The conjecture was substantiated by Boggio's explicit formula [6] (see also [15, Lemma 2.27] or [28]) for the Greens function of problem (1.1) on the unit disc, because this function is positive, and furthermore by Almansi's result to calculate the Greens function for certain domains by the Greens function of the unit ball (see [3]).

Sascha Eichmann

sascha.eichmann@math.uni-tuebingen.de

Reiner M. Schätzle

schaetz@everest.mathematik.uni-tuebingen.de

1 Fachbereich Mathematik der Eberhard-Karls-Universität Tübingen, Auf der Morgenstelle 10, 72076 Tübingen, Germany 
Several other domains than the disc have been found on which such a positivity preserving property holds (see the references below). Hadamard himself claimed in [27] that such a property for all limaçons is true, which turned out to be wrong in general, though some of them still possess this property (see [15, Fig. 1.2]). Remarkable is that such limaçons are not convex. In [20], Grunau and Robert showed that positivity preserving is preserved under small regular perturbations of the domain in dimensions $n \geq 3$.

The conformal invariance of the problem was also successfully used to construct domains with a positivity preserving property by, e.g. Dall'Acqua and Sweers in [10] (see also the references therein for more information on such domains).

On the other hand, several counterexamples have been found by now. The first one was by Duffin on an infinite strip [12] and shortly after Garabedian [14] showed that on an elongated ellipse the Greens function changes sign. By now even for uniform forces, i.e. $f \equiv 1$, counterexamples have been found by Grunau and Sweers in [23] and [24]. We refer to [15, Sect. 1.2] for a comprehensive historical overview to this problem.

Instead of examining (1.1) for positivity, Cassani and Tarsia in [9] examined positivity issues for

$$
\left\{\begin{array}{cc}
\Delta^{2} u-\gamma \Delta u=f & \text { in } \Omega, \\
u=\partial_{\nu} u=0 & \text { on } \partial \Omega .
\end{array}\right.
$$

with $\gamma>0$ big enough. The basic motivation is that for $\gamma$ big enough, the influence of $\Delta u$ $(-\Delta u=f$ satisfies positivity preserving via the maximum principle) becomes stronger than that of $\Delta^{2} u$. In more technical detail Cassani and Tarsia conjectured the existence of a $\gamma_{0}=\gamma_{0}(f, \Omega) \geq 0$, such that $u \geq 0$ for all $\gamma \geq \gamma_{0}$ and provided a proof for dimensions $n=2,3$, smooth, bounded $\Omega$ and positive $f \in L^{2}(\Omega)$. In this article we provide a different approach, which is valid for all dimensions, see Theorem 1.1.

In dimension $n=1$, this positivity preserving property is true for all $\gamma>0$ independent of $f$. This was shown by Grunau in [19] Proposition 1 .

The parameter $\gamma$ is usually called tension, if it is positive. Several results concerning (1.2) have been achieved, which are usually concerned with vibrations of the plate, i.e. eigenvalue problems. Bickley gave some explicit calculations for the spectrum in the unit disc in [5] already in 1933. Hence the existing literature for these eigenvalue problems is quite vast and is still developing, see, e.g. [8, 4] and the references therein.

Other modifications for (1.1) concerning positivity issues are, e.g. changing the boundary conditions to so called Steklov conditions. This has been examined by, e.g. Bucur and Gazzola in [7].

Different elliptic differential operators of higher order, their respective fundamental solutions and their sign close to a singularity have also been examined by Grunau, Romani and Sweers in [22] in a more systematic approach to understand better the loss of positivity preserving.

Instead of (1.2), we examine the following boundary value problem for positivity preserving. This is obviously equivalent, but (1.3) yields the advantage, that the singularity of the equation is more prominent and hence yields easier access to necessary estimates.

$$
\left\{\begin{array}{cc}
\varepsilon^{2} \Delta^{2} u_{\varepsilon}-\Delta u_{\varepsilon}=f & \text { in } \Omega, \\
u_{\varepsilon}=\partial_{\nu} u_{\varepsilon}=0 & \text { on } \partial \Omega .
\end{array}\right.
$$


Here $\quad \Omega \subset \subset \mathbb{R}^{n}, \quad \partial \Omega \in C^{4}, \quad f \in L^{\infty}(\Omega) \quad$ and $\quad \varepsilon>0, \quad$ and the solution $u_{\varepsilon} \in W^{4, p}(\Omega)$ for all $1<p<\infty$ (see, e.g. [15, Corollary 2.21] and the references therein for existence, regularity and uniqueness to (1.3)).

Theorem 1.1 For connected $\Omega \subset \subset \mathbb{R}^{n}, \partial \Omega \in C^{4}, f \in L^{\infty}(\Omega), \tau>0, f \geq 0$ with

$$
\int f \mathrm{~d} \mathcal{L}^{n} \geq \tau\|f\|_{L^{\infty}(\Omega)}>0,
$$

there exists $\varepsilon_{0}=\varepsilon_{0}(\Omega, \tau)>0$ such that

$$
u_{\varepsilon}>0 \text { in } \Omega \text { for all } 0<\varepsilon \leq \varepsilon_{0} .
$$

Please note that we do not have any restrictions on the dimension, i.e. $n \in \mathbb{N}$ arbitrary. Furthermore, our method yields that $\varepsilon_{0}$ does not depend on $f$ directly, only on $\tau$.

The strategy of the proof is as follows: The limiting problem of (1.3) is

$$
\left\{\begin{array}{cc}
-\Delta u=f \geq 0, \not \equiv 0 & \text { in } \Omega, \\
u=0 & \text { on } \partial \Omega,
\end{array}\right.
$$

which admits a maximum principle, and establishes positivity of $u_{\varepsilon}$ on any $\Omega^{\prime} \subset \subset \Omega$ for $\varepsilon$ small.

We proceed by contradiction and assume Theorem 1.1 is false. Hence for every $\varepsilon>0$, we find a nonnegative $f_{\varepsilon} \in L^{\infty}(\Omega)$ satisfying (1.4), such that $u_{\varepsilon}$ is not positive in $\Omega$.

Then, we examine a blow-up of our solutions $u_{\varepsilon}$, which is weighted by the supremum of the modulus of the Laplacian at the boundary, i.e. $\sup _{\partial \Omega} \varepsilon^{2}\left|\Delta u_{\varepsilon}\right|$. After a careful analysis (see Sects. 2 and 3), we can show that this blow-up converges in a suitable sense to a solution of $\Delta^{2} u-\Delta u=0$ on the half-space with Dirichlet boundary conditions (see Sect. 4). With a uniqueness result shown in Appendix A, we explicitly calculate this limit and obtain positivity of the Laplacian of $u_{\varepsilon}$ on the boundary for $\varepsilon$ small. This is crucial, as in the presence of Dirichlet boundary conditions in (1.3) the Laplacian is the second normal derivative of $u_{\varepsilon}$ on the boundary, and therefore positivity of the Laplacian on the boundary gives positivity of $u_{\varepsilon}$ close to the boundary, see Sect. 4.

Similar strategies of examining a blow-up to the half space and using explicit formulas have been employed by Grunau and Robert in [20] and Grunau, Robert and Sweers in [21] to show lower bounds for the Greens function of a polyharmonic operator. This method was later refined by Pulst in his PhD-thesis [30] to also obtain such estimates, if non-constant lowerorder terms are present. If variable coefficients in the principal part of the operator are given by a power of a second-order elliptic linear operator, such estimates were found by the same method by Grunau in [18].

Our blow-up strategy needs careful estimates for the singular problem (1.3). Estimates for these kinds of problems have a long history, see, e.g. [13, 17, 25] and [29]. We are not aware of any specific estimates, which would help in our specific situation. For this reason and for the sake of completeness, we derive them here. 


\section{Preliminary estimates}

We proceed by contradiction and assume we find nonnegative $f_{\varepsilon} \in L^{\infty}(\Omega)$ for $\varepsilon \downarrow 0$ satisfying (1.4), such that the solution $u_{\varepsilon}$ of (1.3) is sign-changing. As (1.3) is homogeneous of degree one, we may assume by scaling

$$
0 \leq f_{\varepsilon} \leq 1 \quad \text { and } \quad \int f_{\varepsilon} \mathrm{d} \mathcal{L}^{n} \geq \tau>0
$$

By the Banach-Alaoglu Theorem, we get after passing to subsequence and relabelling $f_{\varepsilon} \rightarrow f$ weakly* in $L^{\infty}(\Omega)$ with

$$
0 \leq f \leq 1 \quad \text { and } \quad \int f \mathrm{~d} \mathcal{L}^{n} \geq \tau>0 .
$$

In particular, we have $f \not \equiv 0$.

The limiting problem of (1.3) is thought to be the second-order boundary-value problem

$$
\begin{array}{cc}
-\Delta u=f & \text { in } \Omega, \\
u=0 & \text { on } \partial \Omega .
\end{array}
$$

We also consider

$$
\begin{array}{cc}
-\Delta u_{0, \varepsilon}=f_{\varepsilon} & \text { in } \Omega, \\
u_{0, \varepsilon}=0 & \text { on } \partial \Omega .
\end{array}
$$

These two problems admit some important estimates:

Proposition 2.1 For $u$ and $u_{0, \varepsilon}$ in (2.3) rsp. (2.4) we find a constant $c_{0}=c_{0}(\Omega, \tau)>0$, such that

$$
-\partial_{\nu} u,-\partial_{\nu} u_{0, \varepsilon} \geq c_{0}>0 \quad \text { on } \partial \Omega
$$

and

$$
u, u_{0, \varepsilon} \geq c_{0} d(., \partial \Omega) \quad \text { on } \Omega
$$

for $\varepsilon>0$ small enough.

Proof Both problems admit by standard elliptic theory, see [16] Theorem 9.15, unique solutions $\quad u$ respectively $u_{0, \varepsilon} \in W^{2, p}(\Omega) \hookrightarrow C^{1, \alpha}(\Omega)$ for all $1<p<\infty$ and $1-(n / p)>\alpha>0$ with

$$
\left\|u, u_{0, \varepsilon}\right\|_{W^{2, p}(\Omega) \cap C^{1, \alpha}(\Omega)} \leq C(\Omega, p, \alpha)<\infty \quad \forall 1<p<\infty, 0<\alpha<1 .
$$

In particular, the set of all $u, u_{0, \varepsilon}$ for any $f, f_{\varepsilon}$ with (2.2) and (2.1) is compact in $C^{1}(\bar{\Omega})$, and in particular

$$
u_{0, \varepsilon} \rightarrow u \quad \text { strongly in } C^{1, \alpha}(\Omega), \forall 0<\alpha<1,
$$

and weakly in $W^{2, p}(\Omega)$ for all $1<p<\infty$.

As $f, f_{\varepsilon} \geq 0, f, f_{\varepsilon} \not \equiv 0$ and $\Omega$ is connected, we get by the strong maximum principle, see [16] Theorem 8.19, that 


$$
u, u_{0, \varepsilon}>0 \text { in } \Omega \text {. }
$$

By Hopf's maximum principle, see [16] Lemma 3.4, and by compactness of the $u, u_{0, \varepsilon}$ in $C^{1}(\bar{\Omega})$ we find a constant $c_{0}=c_{0}(\Omega, \tau)>0$ such that (2.5) and (2.6) both hold for $\varepsilon>0$ small enough.

We put $v_{\varepsilon}:=u_{\varepsilon}-u_{0, \varepsilon} \in W^{2, p}(\Omega) \cap C^{1, \alpha}(\Omega)$ with $v_{\varepsilon}=0$ on $\partial \Omega$. Subtracting in (1.3), we see

$$
\Delta\left(\varepsilon^{2} \Delta u_{\varepsilon}-v_{\varepsilon}\right)=0 \quad \text { in } \Omega,
$$

that is $\varepsilon^{2} \Delta u_{\varepsilon}-v_{\varepsilon}$ is harmonic in $\Omega$.

Equation (2.3) is indeed the limiting problem of (1.3) in the sense of the following proposition.

Proposition 2.2 For $u_{\varepsilon}, u, v_{\varepsilon}=u_{\varepsilon}-u_{0, \varepsilon}$ as in (1.3), (2.1), (2.4), we have

$$
\begin{gathered}
u_{\varepsilon} \rightarrow u, v_{\varepsilon} \rightarrow 0 \text { strongly in } W_{0}^{1,2}(\Omega), \\
\varepsilon \Delta u_{\varepsilon} \rightarrow 0 \text { strongly in } L^{2}(\Omega) .
\end{gathered}
$$

Proof Multiplying (2.10) by $v_{\varepsilon}$ and integrating by parts, we get

$$
\begin{aligned}
0 & =\int_{\Omega} \Delta\left(\varepsilon^{2} \Delta u_{\varepsilon}-v_{\varepsilon}\right) v_{\varepsilon} \mathrm{d} \mathcal{L}^{n} \\
& =\int_{\Omega} \varepsilon^{2}\left(\Delta^{2} u_{\varepsilon}\right) u_{\varepsilon} \mathrm{d} \mathcal{L}^{n}-\int_{\Omega} \varepsilon^{2}\left(\Delta^{2} u_{\varepsilon}\right) u_{0, \varepsilon} \mathrm{d} \mathcal{L}^{n}+\int_{\Omega}\left|\nabla v_{\varepsilon}\right|^{2} \mathrm{~d} \mathcal{L}^{n} .
\end{aligned}
$$

Replacing $\varepsilon^{2} \Delta^{2} u_{\varepsilon}$ by $\Delta v_{\varepsilon}$ in the second term with (2.10), we continue

$$
\begin{gathered}
\int_{\Omega} \varepsilon^{2}\left|\Delta u_{\varepsilon}\right|^{2} \mathrm{~d} \mathcal{L}^{n}+\int_{\Omega}\left|\nabla v_{\varepsilon}\right|^{2} \mathrm{~d} \mathcal{L}^{n}=\int_{\Omega}\left(\Delta v_{\varepsilon}\right) u_{0, \varepsilon} \mathrm{d} \mathcal{L}^{n} \\
=-\int_{\Omega} \nabla v_{\varepsilon} \nabla u_{0, \varepsilon} \mathrm{d} \mathcal{L}^{n} \leq\left\|\nabla v_{\varepsilon}\right\|_{L^{2}(\Omega)}\left\|\nabla u_{0, \varepsilon}\right\|_{L^{2}(\Omega)},
\end{gathered}
$$

in particular $\left\|\nabla v_{\varepsilon}\right\|_{L^{2}(\Omega)} \leq\left\|\nabla u_{0, \varepsilon}\right\|_{L^{2}(\Omega)} \leq C(\Omega)$ by $(2.7)$, hence

$$
\int_{\Omega} \varepsilon^{2}\left|\Delta u_{\varepsilon}\right|^{2} \mathrm{~d} \mathcal{L}^{n}+\int_{\Omega}\left|\nabla v_{\varepsilon}\right|^{2} \mathrm{~d} \mathcal{L}^{n} \leq \int_{\Omega}\left|\nabla u_{0, \varepsilon}\right|^{2} \mathrm{~d} \mathcal{L}^{n} \leq C(\Omega) .
$$

Passing to a subsequence, we get

$$
\begin{gathered}
v_{\varepsilon} \rightarrow v \text { weakly in } W_{0}^{1,2}(\Omega), \\
\varepsilon^{2} \Delta u_{\varepsilon} \rightarrow 0 \text { strongly in } L^{2}(\Omega) .
\end{gathered}
$$

Multiplying (2.10) by some $\eta \in C_{0}^{\infty}(\Omega)$ and passing to a subsequence, we get

$$
0=\int_{\Omega}\left(\varepsilon^{2} \Delta^{2} u_{\varepsilon}-\Delta v_{\varepsilon}\right) \eta \mathrm{d} \mathcal{L}^{n}=\int_{\Omega} \varepsilon^{2} \Delta u_{\varepsilon} \cdot \Delta \eta \mathrm{d} \mathcal{L}^{n}+\int_{\Omega} \nabla v_{\varepsilon} \nabla \eta \mathrm{d} \mathcal{L}^{n} \rightarrow \int_{\Omega} \nabla v \nabla \eta \mathrm{d} \mathcal{L}^{n},
$$


and $v \in W_{0}^{1,2}(\Omega)$ is harmonic in $\Omega$, hence $v=0$ and $u_{\varepsilon} \rightarrow u$ weakly in $W_{0}^{1,2}(\Omega)$. Returning to (2.12), we improve now to

$$
\int_{\Omega} \varepsilon^{2}\left|\Delta u_{\varepsilon}\right|^{2} \mathrm{~d} \mathcal{L}^{n}+\int_{\Omega}\left|\nabla v_{\varepsilon}\right|^{2} \mathrm{~d} \mathcal{L}^{n}=-\int_{\Omega} \nabla v_{\varepsilon} \nabla u_{0, \varepsilon} \mathrm{d} \mathcal{L}^{n} \rightarrow 0,
$$

which is (2.11).

The following proposition shows that the Laplacian cannot be bounded throughout $\Omega$.

Proposition 2.3 For $u_{\varepsilon}, \tau$ as in (1.3), (2.1), (2.2), we have for any $\Omega^{\prime} \subset \subset \Omega$ that

$$
\liminf _{\varepsilon \rightarrow 0} \int_{\Omega \backslash \Omega^{\prime}}\left(\Delta u_{\varepsilon}\right)_{+} \mathrm{d} \mathcal{L}^{n} \geq \tau>0 .
$$

Proof We see for any $\eta \in C_{0}^{\infty}(\Omega)$ with the previous Proposition 2.2

$$
\int\left(\Delta u_{\varepsilon}\right) \eta \mathrm{d} \mathcal{L}^{n}=\int u_{\varepsilon} \Delta \eta \mathrm{d} \mathcal{L}^{n} \rightarrow \int u \Delta \eta \mathrm{d} \mathcal{L}^{n}=\int(\Delta u) \eta \mathrm{d} \mathcal{L}^{n}=-\int f \eta \mathrm{d} \mathcal{L}^{n}
$$

and the homogeneous boundary conditions in (1.3) that

$$
\begin{aligned}
\int & \Delta u_{\varepsilon}(1-\eta) \mathrm{d} \mathcal{L}^{n}=\int \Delta u_{\varepsilon} \mathrm{d} \mathcal{L}^{n}-\int\left(\Delta u_{\varepsilon}\right) \eta \mathrm{d} \mathcal{L}^{n} \\
= & \int_{\partial \Omega} \partial_{\nu} u_{\varepsilon} \mathrm{d} \operatorname{area}_{\partial \Omega}-\int\left(\Delta u_{\varepsilon}\right) \eta \mathrm{d} \mathcal{L}^{n} \rightarrow \int f \eta \mathrm{d} \mathcal{L}^{n} .
\end{aligned}
$$

Choosing $\eta \in C_{0}^{\infty}(\Omega)$ with $0 \leq \eta \leq 1$ and $\eta \equiv 1$ in $\Omega^{\prime}$, we get

$$
\int_{\Omega \backslash \Omega^{\prime}}\left(\Delta u_{\varepsilon}\right)_{+} \mathrm{d} \mathcal{L}^{n} \geq \int\left(\Delta u_{\varepsilon}\right)(1-\eta) \mathrm{d} \mathcal{L}^{n} \rightarrow \int f \eta \mathrm{d} \mathcal{L}^{n} .
$$

Letting $\eta \nearrow \chi_{\Omega}$, we get from (2.2) that

$$
\int f \eta \mathrm{d} \mathcal{L}^{n} \rightarrow \int f \mathrm{~d} \mathcal{L}^{n} \geq \tau>0
$$

and the proposition follows.

\section{The Laplacian on the boundary}

In this section, we investigate the values of the Laplacian on the boundary and put

$$
\ell_{\varepsilon}^{-}:=\min _{\partial \Omega} \varepsilon^{2} \Delta u_{\varepsilon} \leq \max _{\partial \Omega} \varepsilon^{2} \Delta u_{\varepsilon}=: \ell_{\varepsilon}^{+} .
$$

With subscripts \pm , we denote the positive, respectively, negative part, i.e. 


$$
\ell_{\varepsilon,+}^{ \pm}:=\max \left(0, \ell_{\varepsilon}^{ \pm}\right), \ell_{\varepsilon,-}^{ \pm}:=\max \left(0,-\ell_{\varepsilon}^{ \pm}\right)
$$

Furthermore, we set

$$
M_{\varepsilon}:=\left\|\varepsilon^{2} \Delta u_{\varepsilon}\right\|_{L^{\infty}(\partial \Omega)}=\max \left(\ell_{\varepsilon,+}^{+}, \ell_{\varepsilon,-}^{-}\right) .
$$

The quantity $M_{\varepsilon}$ will be crucial throughout the exposition. Our goal is to show that it has the same asymptotic as $\varepsilon$ itself, i.e. we find constants $c_{0}, C>0$ such that

$$
c_{0} \varepsilon \leq M_{\varepsilon} \leq C \varepsilon \text {. }
$$

for $\varepsilon>0$ small. A first step in this direction is Proposition 3.3, which will later be improved to our desired result in Proposition 4.2 and (4.19).

With the maximum principle, we get the following estimates.

Proposition 3.1 For $u_{\varepsilon}, f_{\varepsilon}, u_{0, \varepsilon}, v_{\varepsilon}=u_{\varepsilon}-u_{0, \varepsilon}, \ell_{\varepsilon}^{ \pm}$as in (1.3), (2.1), (2.4), (3.1), we have

$$
\begin{gathered}
\ell_{\varepsilon}^{-} \leq \varepsilon^{2} \Delta u_{\varepsilon}-v_{\varepsilon} \leq \ell_{\varepsilon}^{+} \quad \text { in } \Omega, \\
-\ell_{\varepsilon,+}^{+}-\varepsilon^{2}\left\|f_{\varepsilon}\right\|_{L^{\infty}(\Omega)} \leq v_{\varepsilon} \leq \ell_{\varepsilon,-}^{-} \quad \text { in } \Omega, \\
u_{\varepsilon}>-\ell_{\varepsilon,+}^{+} \quad \text { in } \Omega .
\end{gathered}
$$

Proof As $v_{\varepsilon}=u_{\varepsilon}-u_{0, \varepsilon}=0$ on $\partial \Omega$ by (1.3) and (2.4), we get (3.4) from (2.10).

Adding (2.4), we see

$$
\varepsilon^{2} \Delta v_{\varepsilon}-v_{\varepsilon}=-\varepsilon^{2} \Delta u_{0, \varepsilon}+\varepsilon^{2} \Delta u_{\varepsilon}-v_{\varepsilon} \leq \varepsilon^{2} f_{\varepsilon}+\ell_{\varepsilon}^{+} \quad \text { in } \Omega,
$$

in particular

$$
\varepsilon^{2} \Delta v_{\varepsilon}<0 \quad \text { in }\left[v_{\varepsilon}<-\varepsilon^{2} f_{\varepsilon}-\ell_{\varepsilon,+}^{+}\right]=: \Omega_{0} .
$$

Since $v_{\varepsilon} \geq-\varepsilon^{2} f_{\varepsilon}-\ell_{\varepsilon,+}^{+}$on $\partial \Omega_{0}$, as $v_{\varepsilon}=0$ on $\partial \Omega$ by above, we get from the meanvalue estimate for superharmonic functions or by Alexandroff's maximum principle, as $v_{\varepsilon} \in W^{2, n}(\Omega)$, see [16] Theorem 9.1, that $v_{\varepsilon} \geq-\varepsilon^{2} f_{\varepsilon}-\ell_{\varepsilon,+}^{+}$in $\Omega_{0}$, hence $\Omega_{0}=\emptyset$, and the left estimate in (3.5) follows. The right estimate is obtained by symmetry observing that $f_{\varepsilon} \geq 0$.

Next for $x \in \bar{\Omega}$ with $u_{\varepsilon}(x)=\min _{\bar{\Omega}} u_{\varepsilon}$ and assuming that this minimum is negative, we see $x \in \Omega$, as $u_{\varepsilon}=0$ on $\partial \Omega$ by (1.3), hence $\Delta u_{\varepsilon}(x) \geq 0$, as $u_{\varepsilon} \in W^{4, p}(\Omega) \hookrightarrow C^{2}(\Omega)$ for $2-(n / p)>0$. Then, we get with (3.4) and (2.9) that

$$
-u_{\varepsilon}(x) \leq \varepsilon^{2} \Delta u_{\varepsilon}(x)-v_{\varepsilon}(x)-u_{0, \varepsilon}(x)<\ell_{\varepsilon}^{+},
$$

which is (3.6).

Using the fourth-order equation, we get estimates for the Laplacian.

Proposition 3.2 For $u_{\varepsilon}, f_{\varepsilon}, u_{0, \varepsilon}, v_{\varepsilon}=u_{\varepsilon}-u_{0, \varepsilon}, \ell_{\varepsilon}^{ \pm}$as in (1.3), (2.1), (2.4), (3.1), we have 


$$
-\ell_{\varepsilon,-}^{-}-\varepsilon^{2}\left\|f_{\varepsilon}\right\|_{L^{\infty}(\Omega)} \leq \varepsilon^{2} \Delta u_{\varepsilon} \leq \ell_{\varepsilon,+}^{+} \quad \text { in } \Omega .
$$

Proof We have with (1.3) that

$$
\varepsilon^{2} \Delta^{2} u_{\varepsilon}-\Delta u_{\varepsilon}=f_{\varepsilon} \quad \text { in } \Omega
$$

and get

$$
\varepsilon^{2} \Delta\left(\Delta u_{\varepsilon}\right)<0 \quad \text { in }\left[\Delta u_{\varepsilon}<-\left\|f_{\varepsilon,+}\right\|_{L^{\infty}(\Omega)}-\varepsilon^{-2} \ell_{\varepsilon,-}^{-}\right]=: \Omega_{0} .
$$

Since $\quad \Delta u_{\varepsilon} \geq-\left\|f_{\varepsilon,+}\right\|_{L^{\infty}(\Omega)}-\varepsilon^{-2} \ell_{\varepsilon,-}^{-}$on $\partial \Omega_{0}, \quad$ as $\quad \Delta u_{\varepsilon} \geq \varepsilon^{-2} \ell_{\varepsilon}^{-} \geq-\varepsilon^{-2} \ell_{\varepsilon,-}^{-}$on $\partial \Omega$ with (3.1), we get from the mean-value estimate for superharmonic functions or by Alexandroff's maximum principle, as $\Delta u_{\varepsilon} \in W^{2, n}(\Omega)$, see [16] Theorem 9.1, that $\Delta u_{\varepsilon} \geq-\left\|f_{\varepsilon,+}\right\|_{L^{\infty}(\Omega)}-\varepsilon^{-2} \ell_{\varepsilon,-}^{-}$in $\Omega_{0}$, hence $\Omega_{0}=\emptyset$, and the left estimate in (3.7) follows. The right estimate is obtained by symmetry observing that $f_{\varepsilon} \geq 0$.

Here, we can give a preliminary asymptotic estimate for $M_{\varepsilon}$. Actually, we will improve this asymptotic later in Proposition 4.2 and (4.19). Anyway we present this estimate at this stage to get more compact bounds already now.

Proposition 3.3 For $u_{\varepsilon}, M_{\varepsilon}, \ell_{\varepsilon}^{ \pm}$as in (1.3), (2.1), (3.1), (3.3), we have

$$
\varepsilon^{-2} M_{\varepsilon} \geq \varepsilon^{-2} \ell_{\varepsilon,+}^{+} \rightarrow \infty \text {. }
$$

in particular $M_{\varepsilon} \geq \ell_{\varepsilon}^{+}=\ell_{\varepsilon,+}^{+}>0$ for $\varepsilon$ small depending on $\Omega$ and $\tau$.

Proof Combining Proposition 3.2 (3.7) and Proposition 2.3, we get for any $\Omega^{\prime} \subset \subset \Omega$ that

$$
\mathcal{L}^{n}\left(\Omega \backslash \Omega^{\prime}\right) \liminf _{\varepsilon \rightarrow 0} \varepsilon^{-2} \ell_{\varepsilon,+}^{+} \geq \liminf _{\varepsilon \rightarrow 0} \int_{\Omega \backslash \Omega^{\prime}}\left(\Delta u_{\varepsilon}\right)_{+} \mathrm{d} \mathcal{L}^{n} \geq \tau>0,
$$

hence, as $\mathcal{L}^{n}\left(\Omega \backslash \Omega^{\prime}\right)$ can be made arbitrarily small, that

$$
\varepsilon^{-2} \ell_{\varepsilon,+}^{+} \rightarrow \infty
$$

which yields the assertion.

With the above asymptotic, we can already bound $v_{\varepsilon}$, and we can prove that $u$ is positive on large parts of $\Omega$.

Proposition 3.4 For $u_{\varepsilon}, u_{0, \varepsilon}, v_{\varepsilon}=u_{\varepsilon}-u_{0, \varepsilon}, M_{\varepsilon}$ as in (1.3), (2.1), (2.2), (2.4), (3.3), we have

$$
\limsup _{\varepsilon \rightarrow 0}\left\|M_{\varepsilon}^{-1} v_{\varepsilon}\right\|_{L^{\infty}(\Omega)} \leq 1 \text {. }
$$

Proof Combining Proposition 3.1 (3.5) and Proposition 3.3, we get observing (2.1) that

$$
\limsup _{\varepsilon \rightarrow 0}\left\|M_{\varepsilon}^{-1} v_{\varepsilon}\right\|_{L^{\infty}(\Omega)} \leq \limsup _{\varepsilon \rightarrow 0} M_{\varepsilon}^{-1}\left(M_{\varepsilon}+\varepsilon^{2}\left\|f_{\varepsilon}\right\|_{L^{\infty}(\Omega)}\right) \leq 1 .
$$


Proposition 3.5 For $u_{\varepsilon}, \ell_{\varepsilon}^{ \pm}$as in (1.3), (2.1), (3.1), we have

$$
u_{\varepsilon}>0 \quad \text { in }\left[d(., \partial \Omega) \geq C \ell_{\varepsilon}^{+}\right] \cap \Omega
$$

for some $C=C(\Omega, \tau)<\infty$ and $\varepsilon$ small depending on $\Omega$ and $\tau$.

Proof From (2.6), we see for $x \in \Omega$ with $c_{0} d(x, \partial \Omega)>\ell_{\varepsilon,+}^{+}+\varepsilon^{2}\left\|f_{\varepsilon}\right\|_{L^{\infty}(\Omega)}$ by (3.5) that

$$
u_{\varepsilon}(x)=v_{\varepsilon}(x)+u(x) \geq-\ell_{\varepsilon,+}^{+}-\varepsilon^{2}\left\|f_{\varepsilon}\right\|_{L^{\infty}(\Omega)}+c_{0} d(x, \partial \Omega)>0 .
$$

By Proposition 3.3 and (2.1) clearly

$$
2 \ell_{\varepsilon}^{+} \geq \ell_{\varepsilon,+}^{+}+\varepsilon^{2}\left\|f_{\varepsilon}\right\|_{L^{\infty}(\Omega)}
$$

for $\varepsilon$ small, and (3.8) follows for $C=2 c_{0}^{-1}<\infty$.

\section{Blow-up}

In this section, we consider a blow-up of our solutions $u_{\varepsilon}$ by translating and rescaling with $x_{0, \varepsilon} \in \mathbb{R}^{n}$. We will have to choose $x_{0, \varepsilon}$ differently in different steps in the proof of Theorem 1.1. In, e.g. the proof of Claim 1 in the proof of Theorem 1.1, we choose $x_{0, \varepsilon} \in \partial \Omega$ such that $\max _{x \in \partial \Omega}\left|\varepsilon^{2} \Delta u_{\varepsilon}(x)\right|$ is attained, while in the proof of Claim 2, we choose $x_{0, \varepsilon} \in \partial \Omega$ to attain $\ell_{\varepsilon}^{-}$. This will not cause a problem, because the constants yielded by these claims do not depend on $\varepsilon$. We put

$$
\tilde{u}_{\varepsilon}(x):=u_{\varepsilon}\left(x_{0, \varepsilon}+\varepsilon x\right) \quad \text { for } x \in \tilde{\Omega}_{\varepsilon}:=\varepsilon^{-1}\left(\Omega-x_{0, \varepsilon}\right) .
$$

Then

$$
\nabla \tilde{u}_{\varepsilon}=\varepsilon \nabla u_{\varepsilon}\left(x_{0, \varepsilon}+\varepsilon .\right), \quad \Delta \tilde{u}_{\varepsilon}=\varepsilon^{2} \Delta u_{\varepsilon}\left(x_{0, \varepsilon}+\varepsilon .\right)
$$

and by (1.3) that

$$
\begin{aligned}
\Delta^{2} \tilde{u}_{\varepsilon}-\Delta \tilde{u}_{\varepsilon}=\varepsilon^{2} f_{\varepsilon}\left(x_{0, \varepsilon}+\varepsilon .\right)=: \tilde{f}_{\varepsilon} & \text { on } \tilde{\Omega}_{\varepsilon}, \\
\tilde{u}_{\varepsilon}, \partial_{v_{\tilde{\Omega}_{\varepsilon}}} \tilde{u}_{\varepsilon}=0 & \text { on } \partial \tilde{\Omega}_{\varepsilon}
\end{aligned}
$$

and with (2.1) and Proposition 3.3 that

$$
\left\|\tilde{f}_{\varepsilon}\right\|_{L^{\infty}\left(\tilde{\Omega}_{\varepsilon}\right)}=\left\|f_{\varepsilon}\right\|_{L^{\infty}(\Omega)} \varepsilon^{2}=o(1) M_{\varepsilon} \quad \text { for } \varepsilon \rightarrow 0,
$$

where $o(1) \rightarrow 0$ depending on $\Omega$ and $\tau$. We extend $u_{\varepsilon}$ respectively $\tilde{u}_{\varepsilon}$ by putting 0 outside $\Omega$ respectively outside $\tilde{\Omega}_{\varepsilon}$. By the homogeneous boundary conditions in (1.3) and (4.3), we see $u_{\varepsilon}, \tilde{u}_{\varepsilon} \in W_{l o c}^{2,2}\left(\mathbb{R}^{n}\right)$.

We also have to stretch $\tilde{u}_{\varepsilon}$ to get a nontrivial limit, and it turns out that reaching bounded values of the Laplacian of $\tilde{u}_{\varepsilon}$ on the boundary is the right measure for stretching.

Proposition 4.1 For $\tilde{u}_{\varepsilon}, \tilde{f}_{\varepsilon}, u_{\varepsilon}, f_{\varepsilon}, M_{\varepsilon}$ as in (4.1), (1.3), (2.1), (3.3) and $x_{0, \varepsilon} \in \partial \Omega$, we get for any subsequence with after rotating $\tilde{\Omega}_{\varepsilon}$ such that 


$$
v_{\Omega}\left(x_{0, \varepsilon}\right) \rightarrow-e_{n}
$$

after passing to a subsequence

$$
\begin{gathered}
M_{\varepsilon}^{-1} \tilde{u}_{\varepsilon} \rightarrow \tilde{u}_{\infty} \quad \text { weakly in } W^{2,2}\left(B_{R}(0)\right) \text { for all } R>0, \\
M_{\varepsilon}{ }^{-1} \tilde{u}_{\varepsilon} \rightarrow \tilde{u}_{\infty} \quad \text { weakly in } W^{4, p}\left(B_{R}(0) \cap \tilde{\Omega}_{\varepsilon}\right) \text { for all } R>0,1<p<\infty
\end{gathered}
$$

as $\varepsilon \rightarrow 0$ after flattening the boundary of $\partial \tilde{\Omega}_{\varepsilon}$. Further

$$
\begin{gathered}
\Delta^{2} \tilde{u}_{\infty}-\Delta \tilde{u}_{\infty}=0 \quad \text { in } \mathbb{R}_{+}^{n}, \\
\tilde{u}_{\infty}, \partial_{n} \tilde{u}_{\infty}=0 \quad \text { on } \mathbb{R}^{n-1} \times\{0\}, \\
\tilde{u}_{\infty}=0 \quad \text { in } \mathbb{R}^{n} \backslash \mathbb{R}_{+}^{n}, \\
\tilde{u}_{\infty} \geq-1, \quad\left|\Delta \tilde{u}_{\infty}\right| \leq 1 \quad \text { in } \mathbb{R}^{n} .
\end{gathered}
$$

Proof We get from Proposition 3.1 (3.6) that

$$
\tilde{u}_{\varepsilon} \geq-M_{\varepsilon} \quad \text { in } \tilde{\Omega}_{\varepsilon}
$$

and also outside $\tilde{\Omega}_{\varepsilon}$, as $\tilde{u}_{\varepsilon}=0$ there. Next by Proposition 3.2 (3.7), Proposition 3.3, (2.1) and (4.2) that

$$
\left\|\Delta \tilde{u}_{\varepsilon}\right\|_{L^{\infty}\left(\tilde{\Omega}_{\varepsilon}\right)}=\left\|\varepsilon^{2} \Delta u_{\varepsilon}\right\|_{L^{\infty}(\Omega)} \leq M_{\varepsilon}+\varepsilon^{2}\left\|f_{\varepsilon}\right\|_{L^{\infty}(\Omega)} \leq(1+o(1)) M_{\varepsilon} .
$$

Then, $\tilde{u}_{\varepsilon}+M_{\varepsilon} \geq 0$ in $\mathbb{R}^{n}$, and we can apply the Harnack inequality, see [16] Theorems 8.17 and 8.18 , and get observing that $\tilde{u}_{\varepsilon}(0)=0$, as $x_{0, \varepsilon} \in \partial \Omega$, that

$$
\sup _{B_{R}(0)}\left(\tilde{u}_{\varepsilon}+M_{\varepsilon}\right) \leq C_{n}\left(\tilde{u}_{\varepsilon}+M_{\varepsilon}\right)(0)+C_{n, R}\left\|\Delta\left(\tilde{u}_{\varepsilon}+M_{\varepsilon}\right)\right\|_{L^{\infty}\left(B_{2 R}(0)\right)} \leq C_{n, R} M_{\varepsilon},
$$

hence

$$
\left\|\tilde{u}_{\varepsilon}\right\|_{L^{\infty}\left(B_{R}(0)\right)} \leq C_{n, R} M_{\varepsilon}
$$

for $\varepsilon$ small depending on $\Omega$ and $\tau$.

Next by Friedrichs's Theorem in the interior, see [16] Theorem 8.8, [16] Exercise 8.2, (4.9) and (4.10) that

$$
\left\|\tilde{u}_{\varepsilon}\right\|_{W^{2,2}\left(B_{R}(0)\right)} \leq C_{n, R}\left(\left\|\Delta \tilde{u}_{\varepsilon}\right\|_{L^{2}\left(B_{2 R}(0)\right)}+\left\|\tilde{u}_{\varepsilon}\right\|_{L^{2}\left(B_{2 R}(0)\right)}\right) \leq C_{n, R} M_{\varepsilon}
$$

for $\varepsilon$ small depending on $\Omega$ and $\tau$, which yields the first convergence in (4.6) after passing to a subsequence.

Proceeding from (4.3), we get from fourth-order $L^{p}$-estimates, see [1, 2] Sect. 10, after flattening the boundary of $\partial \tilde{\Omega}_{\varepsilon}$, as $\partial \Omega \in C^{4}$, with (4.4) and (4.10) that

$$
\begin{aligned}
\| & \tilde{u}_{\varepsilon} \|_{W^{4, p}\left(B_{R}(0) \cap \tilde{\Omega}_{\varepsilon}\right)} \\
& \leq C_{n, R, p}\left(\left\|\tilde{f}_{\varepsilon}\right\|_{L^{\infty}\left(B_{2 R}(0) \cap \tilde{\Omega}_{\varepsilon}\right)}+\left\|\tilde{u}_{\varepsilon}\right\|_{L^{\infty}\left(B_{2 R}(0) \cap \tilde{\Omega}_{\varepsilon}\right)}\right) \\
& \leq C_{n, R, p} M_{\varepsilon} \quad \forall R>0,1<p<\infty
\end{aligned}
$$

and $\varepsilon$ small depending on $\Omega$ and $\tau$. After passing to a subsequence, we obtain with (4.5) the second convergence in (4.6).

Finally (4.7) follows from (4.3), (4.4), (4.8), (4.9), when recalling that $\tilde{u}_{\varepsilon}=0$ in $\mathbb{R}^{n} \backslash \Omega_{\varepsilon}$. 
Actually by fourth-order higher-order $L^{p}$-estimates, see $[1,2]$ Sect. 10, we get that the blow-up $\tilde{u}_{\infty}$ is smooth on $\overline{\mathbb{R}_{+}^{n}}$.

Now we are able to give a lower bound for $M_{\varepsilon}$ which improves the asymptotic in Proposition 3.3.

Proposition 4.2 For $u_{\varepsilon}, M_{\varepsilon}$ as in (1.3), (2.1), (3.3), we have

$$
M_{\varepsilon} \geq c_{0} \varepsilon
$$

for some $c_{0}=c_{0}(\Omega, \tau)>0$ and $\varepsilon$ small depending on $\Omega$ and $\tau$.

Proof We see for $x_{\varepsilon} \in \Omega$ with $\left|x_{\varepsilon}-x_{0, \varepsilon}\right|=d\left(x_{\varepsilon}, \partial \Omega\right)=\varepsilon$ for $\varepsilon$ small by (2.6), Proposition 3.4 and by the local boundedness of $\tilde{u}_{\varepsilon}$ in Proposition 4.1, or more directly by (4.10), for $\tilde{x}_{\varepsilon}:=\left(x_{\varepsilon}-x_{0, \varepsilon}\right) / \varepsilon \in \overline{B_{1}(0)}$ that

$$
\begin{aligned}
c_{0} \varepsilon & \leq u_{0, \varepsilon}\left(x_{\varepsilon}\right)=u_{\varepsilon}\left(x_{\varepsilon}\right)-v_{\varepsilon}\left(x_{\varepsilon}\right)=\tilde{u}_{\varepsilon}\left(\tilde{x}_{\varepsilon}\right)-v_{\varepsilon}\left(x_{\varepsilon}\right) \\
& \leq\left\|\tilde{u}_{\varepsilon}\right\|_{L^{\infty}\left(B_{1}(0)\right)}+\left\|v_{\varepsilon}\right\|_{L^{\infty}(\Omega)} \leq\left(C_{n, 1}+o(1)\right) M_{\varepsilon},
\end{aligned}
$$

hence

$$
M_{\varepsilon} \geq c_{0} \varepsilon
$$

for $\varepsilon$ small depending on $\Omega$ and $\tau$.

The blow-up for $u_{0, \varepsilon}$ is rather elementary by the strong convergence in $C^{1, \alpha}(\Omega)$ in (2.8). As in (4.1), we put

$$
\tilde{u}_{0, \varepsilon}(x):=u_{0, \varepsilon}\left(x_{0, \varepsilon}+\varepsilon x\right) \quad \text { for } x \in \tilde{\Omega}_{\varepsilon}:=\varepsilon^{-1}\left(\Omega-x_{0, \varepsilon}\right) .
$$

Proposition 4.3 For $\tilde{u}_{0, \varepsilon}, u_{\varepsilon}, u_{0, \varepsilon}, f_{\varepsilon}, M_{\varepsilon}$ as in (4.12), (1.3), (2.1), (3.3), and with (4.5), we have after passing to a subsequence such that

$$
\beta \leftarrow\left|\nabla u_{0, \varepsilon}\left(x_{0, \varepsilon}\right)\right| \varepsilon / M_{\varepsilon} \geq c_{0} \varepsilon / M_{\varepsilon} \geq 0
$$

for some $c_{0}=c_{0}(\Omega, \tau)>0$ for the linear function $\tilde{u}_{0, \infty}:(y, t) \rightarrow \beta t$ that

$$
M_{\varepsilon}^{-1} \tilde{u}_{0, \varepsilon} \rightarrow \tilde{u}_{0, \infty} \quad \text { uniformly in } B_{R}(0) \cap \tilde{\Omega}_{\varepsilon} \text { for all } R>0
$$

after flattening the boundary of $\partial \tilde{\Omega}_{\varepsilon}$. Further

$$
\left|\tilde{u}_{\infty}-\tilde{u}_{0, \infty}\right| \leq 1 \quad \text { in } \mathbb{R}_{+}^{n},
$$

in particular $\tilde{u}_{\infty}-\tilde{u}_{0, \infty} \in L^{\infty}\left(\mathbb{R}_{+}^{n}\right)$.

Proof From (2.7) and, as $u_{0, \varepsilon}=0$ on $\partial \Omega$ by (2.4), we get by Taylor's expansion for any $x \in \mathbb{R}_{+}^{n}$ and any $0<\alpha<1$ that

$$
M_{\varepsilon}^{-1} \tilde{u}_{0, \varepsilon}(x)=M_{\varepsilon}^{-1} u_{0, \varepsilon}\left(x_{0, \varepsilon}+\varepsilon x\right)=M_{\varepsilon}^{-1} \varepsilon \nabla u_{0, \varepsilon}\left(x_{0, \varepsilon}\right) \cdot x+M_{\varepsilon}^{-1} O_{\alpha}\left(\varepsilon^{1+\alpha}|x|^{1+\alpha}\right) .
$$

As $u_{0, \varepsilon}=0$ on $\partial \Omega$ by (2.4), we get with (2.8), (4.5) and $\partial \Omega \in C^{4}$ after passing to a subsequence with $x_{0, \varepsilon} \rightarrow x_{0} \in \partial \Omega$ that 


$$
\nabla u_{0, \varepsilon}\left(x_{0, \varepsilon}\right)=\partial_{v_{\Omega}} u_{0, \varepsilon}\left(x_{0, \varepsilon}\right) v_{\Omega}\left(x_{0, \varepsilon}\right) \rightarrow-\partial_{\nu_{\Omega}} u\left(x_{0}\right) e_{n},
$$

in particular with (2.5) that

$$
\left|\nabla u_{0, \varepsilon}\left(x_{0, \varepsilon}\right)\right| \rightarrow-\partial_{\nu_{\Omega}} u\left(x_{0}\right)>0
$$

and $\nabla u_{0, \varepsilon}\left(x_{0, \varepsilon}\right) /\left|\nabla u_{0, \varepsilon}\left(x_{0, \varepsilon}\right)\right| \rightarrow e_{n}$. By Proposition 4.2 we extract a subsequence, such that $\varepsilon / M_{\varepsilon}$ converges for $\varepsilon \downarrow 0$. Then, (2.5) yields

$$
\beta:=\leftarrow\left|\nabla u_{0, \varepsilon}\left(x_{0, \varepsilon}\right)\right| \varepsilon / M_{\varepsilon} \geq c_{0} \varepsilon / M_{\varepsilon} \geq 0,
$$

which is (4.13). Furthermore, Proposition 4.2 yields

$$
\varepsilon^{1+\alpha} / M_{\varepsilon} \rightarrow 0
$$

Together, we get

$$
M_{\varepsilon}^{-1} \varepsilon \nabla u_{0, \varepsilon}\left(x_{0, \varepsilon}\right) \rightarrow \beta e_{n},
$$

and the proposed convergence follows from the Taylor expansion (4.15).

Further we get with Proposition 3.4 that

$$
\left|\tilde{u}_{\infty}-\tilde{u}_{0, \infty}\right| \leftarrow\left|M_{\varepsilon}^{-1} \tilde{u}_{\varepsilon}-M_{\varepsilon}^{-1} \tilde{u}_{0, \varepsilon}\right| \leq \limsup _{\varepsilon \rightarrow 0}\left\|M_{\varepsilon}^{-1} v_{\varepsilon}\right\|_{L^{\infty}(\Omega)} \leq 1,
$$

which is (4.14).

By our investigation of half space solutions in Appendix A, Proposition A.3 applied to $\tilde{u}_{\infty}$ and $\tilde{u}_{0, \infty}$ with Proposition 4.1 (4.7) and Proposition 4.3 (4.14) determines $\tilde{u}_{\infty}$ uniquely as the one-dimensional solution and immediately yields the following Proposition.

Proposition 4.4 For $\tilde{u}_{\infty}$ as in Proposition 4.1 and $\beta$ as in Proposition 4.3, we have

$$
\begin{gathered}
\tilde{u}_{\infty} \equiv 0, \quad \text { if } \beta=0, \\
\Delta \tilde{u}_{\infty} \equiv \beta \geq 0 \quad \text { in } \mathbb{R}^{n-1} \times\{0\}
\end{gathered}
$$

and

$$
\tilde{u}_{\infty}>0 \quad \text { in } \mathbb{R}_{+}^{n}, \quad \text { if } \beta>0 .
$$

Now we are able to conclude the proof of Theorem 1.1.

Proof of Theorem 1.1 We consider $u_{\varepsilon}, f_{\varepsilon}, u_{0, \varepsilon}, \ell \frac{ \pm}{\varepsilon}, M_{\varepsilon}$ as in (1.3), (2.1), (2.4), (3.1), (3.3) and $\tilde{u}_{\varepsilon}, \tilde{f}_{\varepsilon}, \tilde{u}_{0, \varepsilon}$ as in (4.1), (4.12) with their blow-ups $\tilde{u}_{\infty}, \tilde{u}_{0, \infty}$ and $\beta$ obtained in the Propositions 4.1 and 4.3. We prove various claims.

\section{Claim 1}

$$
\liminf _{\varepsilon \rightarrow 0} \varepsilon / M_{\varepsilon}>0
$$

hence with Proposition 4.2 that 


$$
c_{0} \leq M_{\varepsilon} / \varepsilon \leq C
$$

for some $c_{0}=c_{0}(\Omega, \tau)>0, C=C(\Omega, \tau)<\infty$ and $\varepsilon$ small depending on $\Omega$ and $\tau$.

Proof If on contrary $\varepsilon / M_{\varepsilon} \rightarrow 0$ for a subsequence $\varepsilon \rightarrow 0$, then we get from Proposition 4.3 and (2.7) that $\beta=0$, hence with Proposition 4.4 (4.16) after passing to this subsequence we have that $\tilde{u}_{\infty} \equiv 0$.

On the other hand, choosing $x_{0, \varepsilon} \in \partial \Omega$ in such a way that

$$
\left|\varepsilon^{2} \Delta u_{\varepsilon}\left(x_{0, \varepsilon}\right)\right|=\left\|\varepsilon^{2} \Delta u_{\varepsilon}\right\|_{L^{\infty}(\partial \Omega)}=M_{\varepsilon},
$$

we get from the convergence in Proposition 4.1 (4.6) that

$$
1=M_{\varepsilon}^{-1}\left|\varepsilon^{2} \Delta u_{\varepsilon}\left(x_{0, \varepsilon}\right)\right|=\left|M_{\varepsilon}^{-1} \Delta \tilde{u}_{\varepsilon}(0)\right| \rightarrow\left|\Delta \tilde{u}_{\infty}(0)\right|,
$$

hence $\Delta \tilde{u}_{\infty}(0) \neq 0$ and $\tilde{u}_{\infty} \not \equiv 0$. This is a contradiction, and the claim follows.

Claim 2

$$
\begin{gathered}
\liminf _{\varepsilon \rightarrow 0} M_{\varepsilon}^{-1} \ell_{\varepsilon}^{-}>0, \\
M_{\varepsilon}^{-1} \Delta \tilde{u}_{\varepsilon} \geq c_{1}>0 \quad \text { on } \partial \tilde{\Omega}_{\varepsilon}
\end{gathered}
$$

for some $c_{1}=c_{1}(\Omega, \tau)>0$ and $\varepsilon$ small depending on $\Omega$ and $\tau$ and

$$
\tilde{u}_{\infty}>0 \quad \text { in } \mathbb{R}_{+}^{n} \text {. }
$$

Proof (4.19) implies with Proposition 4.3 (4.13) that

$$
\beta \geq c_{0} \liminf _{\varepsilon \rightarrow 0} \varepsilon / M_{\varepsilon}>0,
$$

which immediately gives (4.23) by Proposition 4.4 (4.18).

Next we choose $x_{0, \varepsilon} \in \partial \Omega$ in such a way that

$$
\varepsilon^{2} \Delta u_{\varepsilon}\left(x_{0, \varepsilon}\right)=\min _{\partial \Omega} \varepsilon^{2} \Delta u_{\varepsilon}=\ell_{\varepsilon}^{-}
$$

and get as in (4.20) from the convergence in Proposition 4.1 (4.6) and Proposition 4.4 (4.17) that

$$
M_{\varepsilon}^{-1} \ell_{\varepsilon}^{-}=M_{\varepsilon}^{-1} \varepsilon^{2} \Delta u_{\varepsilon}\left(x_{0, \varepsilon}\right)=M_{\varepsilon}^{-1} \Delta \tilde{u}_{\varepsilon}(0) \rightarrow \Delta \tilde{u}_{\infty}(0)=\beta>0,
$$

and (4.21) follows. Clearly (4.21) implies with (3.1) that

$$
M_{\varepsilon}^{-1} \inf _{\partial \tilde{\Omega}_{\varepsilon}} \Delta \tilde{u}_{\varepsilon}=M_{\varepsilon}^{-1} \inf _{\partial \Omega} \varepsilon^{2} \Delta u_{\varepsilon}=M_{\varepsilon}^{-1} \ell_{\varepsilon}^{-} \geq c_{1}>0
$$

for some $c_{1}>0$ and $\varepsilon$ small, which is (4.22).

Further (4.21) implies that $\ell_{\varepsilon}^{+} \geq \ell_{\varepsilon}^{-}>0$ and $\ell_{\varepsilon,-}^{-}=0$ for $\varepsilon$ small, hence with (3.3) that

$$
M_{\varepsilon}=\max \left(\ell_{\varepsilon,+}^{+}, \ell_{\varepsilon,-}^{-}\right)=\ell_{\varepsilon}^{+}
$$

for $\varepsilon$ small depending on $\Omega$ and $\tau$. 


\section{Claim 3}

$$
u_{\varepsilon}>0 \quad \text { in }[d(., \partial \Omega) \geq C \varepsilon] \cap \Omega
$$

for some $C=C(\Omega, \tau)<\infty$ and $\varepsilon$ small depending on $\Omega$ and $\tau$.

This follows directly from Proposition 3.5, (4.19) and (4.24).

\section{Claim 4}

$$
u_{\varepsilon}>0 \quad \text { in }\left[0<d(., \partial \Omega) \leq c_{2} \varepsilon\right] \cap \Omega
$$

for some $c_{2}=c_{2}(\Omega, \tau)>0$ and $\varepsilon$ small depending on $\Omega$ and $\tau$.

By the homogeneous boundary conditions in (1.3), we have $\Delta u_{\varepsilon}\left(x_{0, \varepsilon}\right)=\partial_{\nu \nu} u_{\varepsilon}\left(x_{0, \varepsilon}\right)$, hence with (4.22), Proposition 4.1 (4.6) and the embedding $W^{4, p} \hookrightarrow C^{3}$ for $1-(n / p)>0$ that

$$
\tilde{u}_{\varepsilon}\left(-t v_{\tilde{\Omega}_{\varepsilon}}(0)\right) \geq \frac{1}{2} t^{2} \Delta \tilde{u}_{\varepsilon}(0)-C_{n} M_{\varepsilon} t^{3} \geq \frac{1}{2} t^{2} M_{\varepsilon}\left(c_{1}-2 C_{n} t\right)>0
$$

for $0<t<c_{1} /\left(2 C_{n}\right)<1$ and $\varepsilon$ small. As $x_{0, \varepsilon} \in \partial \Omega$ can be chosen arbitrarily, we get for $c_{2}=c_{1} /\left(4 C_{n}\right)$ that

$$
\tilde{u}_{\varepsilon}>0 \quad \text { in }\left[0<d\left(., \partial \tilde{\Omega}_{\varepsilon}\right) \leq c_{2}\right] \cap \tilde{\Omega}_{\varepsilon},
$$

and the claim follows by rescaling in (4.1).

\section{Claim 5}

$$
u_{\varepsilon}>0 \quad \text { in }\left[c_{2} \varepsilon \leq d(., \partial \Omega) \leq C \varepsilon\right] \cap \Omega
$$

for $\varepsilon$ small depending on $\Omega$ and $\tau$.

Here for any $x_{\varepsilon} \in \Omega$ with $c_{2} \varepsilon \leq d\left(x_{\varepsilon}, \partial \Omega\right) \leq C \varepsilon$ for $\varepsilon$ small, we select $x_{0, \varepsilon} \in \partial \Omega$ with $d\left(x_{\varepsilon}, \partial \Omega\right)=\left|x_{\varepsilon}-x_{0, \varepsilon}\right|$ and get for $\tilde{x}_{\varepsilon}:=\varepsilon^{-1}\left(x_{\varepsilon}-x_{0, \varepsilon}\right) \in \tilde{\Omega}_{\varepsilon}$ that

$$
c_{2} \leq d\left(\tilde{x}_{\varepsilon}, \partial \tilde{\Omega}_{\varepsilon}\right)=\left|\tilde{x}_{\varepsilon}\right| \leq C .
$$

Passing to subsequence, we get $\tilde{x}_{\varepsilon} \rightarrow \tilde{x}$ with $\tilde{x} \in \overline{\mathbb{R}_{+}^{n}}$ and

$$
d\left(\tilde{x}, \mathbb{R}^{n-1} \times\{0\}\right) \geq c_{2}>0,
$$

hence $\tilde{x} \in \mathbb{R}_{+}^{n}$. Then by Proposition 4.1 (4.6) and (4.23) that

$$
M_{\varepsilon}^{-1} u_{\varepsilon}\left(x_{\varepsilon}\right)=M_{\varepsilon}^{-1} \tilde{u}_{\varepsilon}\left(\tilde{x}_{\varepsilon}\right) \rightarrow \tilde{u}_{\infty}(\tilde{x})>0,
$$

and we conclude that $u_{\varepsilon}\left(x_{\varepsilon}\right)>0$ for $\varepsilon$ small, and the claim follows.

Combining (4.25), (4.26) and (4.27), we get

$$
u_{\varepsilon}>0 \quad \text { in } \Omega
$$

for $\varepsilon$ small depending on $\Omega$ and $\tau$, which proves Theorem 1.1. 


\section{Appendix}

\section{Uniqueness of a bi-Laplace equation on the half space}

In this section, we show the following uniqueness theorem.

Theorem A.1 Let $v: \overline{\mathbb{R}_{+}^{n}} \rightarrow \mathbb{R}$ be a smooth solution to

$$
\left\{\begin{array}{c}
\Delta^{2} v-\Delta v=0 \text { on } \mathbb{R}_{+}^{n}, \\
v=\partial_{n} v=0 \text { on } \mathbb{R}^{n-1} \times\{0\}, \\
v \in L^{\infty}\left(\mathbb{R}_{+}^{n}\right) .
\end{array}\right.
$$

Then, $v=0$.

The proof is based on an energy type estimate and on the one-dimensional case. We start with the one-dimensional case and show the following lemma:

Lemma A.1 Let $v \in C_{l o c}^{4}([0, \infty[)$ satisfy

$$
\begin{gathered}
\left.\frac{d^{4} v}{\mathrm{~d} t^{4}}-\frac{d^{2} v}{\mathrm{~d} t^{2}}=0 \text { on }\right] 0, \infty[, \\
v(0)=\frac{\mathrm{d} v}{\mathrm{~d} t}(0)=0, \\
v \in L^{\infty}(] 0, \infty[) .
\end{gathered}
$$

Then, $v=0$.

Proof Since the differential equation is ordinary and linear, the solution space of the equation itself is of dimension 4. By inserting the following functions, we see that they constitute a basis of the solution space

$$
t \mapsto \cosh (t), \sinh (t), 1, t .
$$

The following functions are therefore a basis of the solution space including the initial conditions at $t=0$ :

$$
v_{1}(t)=\cosh (t)-1, v_{2}(t)=\sinh (t)-t .
$$

Hence $v$ has to be of the form

$$
v(t)=A v_{1}(t)+B v_{2}(t)=A(\cosh (t)-1)+B(\sinh (t)-t) .
$$

Inserting the exponential function for cosh and sinh yields

$$
\begin{aligned}
v(t) & =A\left(\frac{e^{t}+e^{-t}}{2}-1\right)+B\left(\frac{e^{t}-e^{-t}}{2}-t\right) \\
& =\frac{1}{2}(A+B) e^{t}+\frac{1}{2}(A-B) e^{-t}-A-B t .
\end{aligned}
$$

Since the solution is bounded, we have $A=-B$. Again the boundedness then yields $B=0$, to rule out linear growth. Hence $v=0$. 
For our next step, we introduce a bit of notation for half spaces

$$
B_{R}^{+}:=B_{R}(0) \cap \mathbb{R}_{+}^{n} \subseteq \mathbb{R}^{n}, R>0 .
$$

Next we show an energy type estimate.

Lemma A.2 Let $v$ satisfy

$$
\left\{\begin{array}{c}
\Delta^{2} v-\Delta v=0 \text { on } \mathbb{R}_{+}^{n}, \\
v=\partial_{n} v=0 \text { on } \mathbb{R}^{n-1} \times\{0\} .
\end{array}\right.
$$

Then, there exists a constant $C=C(n)>0$, such that for all $R>1$ we have

$$
\int_{B_{R}^{+}}\left(|\Delta v|^{2}+|\nabla v|^{2}\right) d \mathcal{L}^{n} \leq \frac{C}{R^{2}} \int_{B_{2 R}^{+}}\left(|\nabla v|^{2}+v^{2}\right) d \mathcal{L}^{n} .
$$

Proof Let $\eta \in C_{0}^{\infty}\left(B_{2 R}(0)\right)$ with $0 \leq \eta \leq 1, \eta=1$ on $B_{R}(0)$ and for any $k \geq 1$

$$
\left|D^{k} \eta\right| \leq \frac{C_{k}}{R^{k}} \chi_{B_{2 R}(0) \backslash B_{R}(0)},
$$

i.e. $\eta$ is a cut-off function for the ball $B_{R}(0)$. Then, $v \eta^{4}$ and its first derivative are zero on $\partial B_{2 R}^{+}$. Therefore, partial integration and the differential equation itself yield

$$
\begin{aligned}
0= & \int_{B_{2 R}^{+}} v \eta^{4}\left(\Delta v-\Delta^{2} v\right) d \mathcal{L}^{n}=-\int_{B_{2 R}^{+}} \Delta\left(v \eta^{4}\right) \Delta v \mathrm{~d} \mathcal{L}^{n}-\int_{B_{2 R}^{+}} \nabla\left(v \eta^{4}\right) \nabla v \mathrm{~d} \mathcal{L}^{n} \\
= & -\int_{B_{2 R}^{+}}\left(|\Delta v|^{2} \eta^{4}+|\nabla v|^{2} \eta^{4}\right) \mathrm{d} \mathcal{L}^{n} \\
& -\int_{B_{2 R}^{+}} 2\left(\nabla v \nabla\left(\eta^{4}\right) \Delta v+v \Delta\left(\eta^{4}\right) \Delta v+v \nabla v \nabla\left(\eta^{4}\right)\right) d \mathcal{L}^{n} \\
= & -\int_{B_{2 R}^{+}}\left(|\Delta v|^{2} \eta^{4}+|\nabla v|^{2} \eta^{4}\right) d \mathcal{L}^{n} \\
& -\int_{B_{2 R}^{+}}\left(8 \nabla v \nabla \eta \eta^{3} \Delta v+4 v \Delta v \Delta \eta \eta^{3}+12 v \Delta v|\nabla \eta|^{2} \eta^{2}+4 v \nabla v \nabla \eta \eta^{3}\right) \mathrm{d} \mathcal{L}^{n} .
\end{aligned}
$$

By the previous identity and Young's inequality with an $\varepsilon>0$ we get

$$
\begin{aligned}
\int_{B_{2 R}^{+}} & \left(|\Delta v|^{2} \eta^{4}+|\nabla v|^{2} \eta^{4}\right) \mathrm{d} \mathcal{L}^{n} \\
\leq & \int_{B_{2 R}^{+}}\left(\varepsilon|\Delta v|^{2} \eta^{4}+C_{\varepsilon}|\nabla v|^{2}|\nabla \eta|^{2} \eta^{2}\right. \\
& +\varepsilon|\Delta v|^{2} \eta^{4}+C_{\varepsilon} v^{2}|\Delta \eta|^{2} \eta^{2} \\
& +\varepsilon|\Delta v|^{2} \eta^{4}+C_{\varepsilon} v^{2}|\nabla \eta|^{4} \\
& \left.+\varepsilon|\nabla v|^{2} \eta^{4}+C_{\varepsilon} v^{2}|\nabla \eta|^{2} \eta^{2}\right) \mathrm{d} \mathcal{L}^{n}
\end{aligned}
$$

Now choosing $\varepsilon$ small enough, we can absorb the terms with $\varepsilon$ as a prefactor into the left hand side 


$$
\begin{aligned}
& \frac{1}{2} \int_{B_{2 R}^{+}}\left(|\Delta v|^{2} \eta^{4}+|\nabla v|^{2} \eta^{4}\right) d \mathcal{L}^{n} \\
& \quad \leq C \int_{B_{2 R}^{+}}\left(|\nabla v|^{2}|\nabla \eta|^{2} \eta^{2}+v^{2}|\Delta \eta|^{2} \eta^{2}+v^{2}|\nabla \eta|^{4}+v^{2}|\nabla \eta|^{2} \eta^{2}\right) d \mathcal{L}^{n}
\end{aligned}
$$

Together with $0 \leq \eta \leq 1$, the estimates on the derivatives of the cut-off function (A.1) yield

$$
\int_{B_{R}^{+}}\left(|\Delta v|^{2}+|\nabla v|^{2}\right) d \mathcal{L}^{n} \leq C \int_{B_{2 R}^{+}}\left(|\nabla v|^{2} \frac{1}{R^{2}}+v^{2} \frac{1}{R^{4}}+v^{2} \frac{1}{R^{4}}+v^{2} \frac{1}{R^{2}}\right) d \mathcal{L}^{n} .
$$

Since we have chosen $R>1$, we obtain

$$
\int_{B_{R}^{+}}\left(|\Delta v|^{2}+|\nabla v|^{2}\right) d \mathcal{L}^{n} \leq \frac{C}{R^{2}} \int_{B_{2 R}^{+}}\left(|\nabla v|^{2}+v^{2}\right) d \mathcal{L}^{n} .
$$

Now we can show our main result Theorem A.1, by iterating Lemma A.2:

Proof of Theorem A.1 Since $v$ is bounded and the differential equation is linear and elliptic, we can employ Schauder-type estimates (see [1, Thm. 6.2] and [11, Sect. 4]) to obtain

$$
\|v\|_{C^{k}\left(\overline{\mathbb{R}_{+}^{n}}\right)} \leq C_{k}\|v\|_{L^{\infty}\left(\overline{\mathbb{R}_{+}^{n}}\right)}<\infty .
$$

Let $x=(y, t) \in \overline{\mathbb{R}_{+}^{n}}$, such that $y \in \mathbb{R}^{n-1}$ and $t \geq 0$. By $\partial_{y}^{k} v$, we denote any partial derivative of $v$ of order $k$ only after horizontal directions, i.e. indices in $\{1, \ldots, n-1\}$. Then, for every $k \in \mathbb{N}$ the function $\partial_{y}^{k} v: \overline{\mathbb{R}_{+}^{n}} \rightarrow \mathbb{R}$ still solves the differential equation, satisfies the Dirichlet boundary conditions and by (A.2) is again bounded. Now we can iteratively apply Lemma A.2 for $k \geq \ell \in \mathbb{N}$ and obtain

$$
\begin{aligned}
& \int_{B_{R}^{+}}\left|\partial_{y}^{k} v\right|^{2} \mathrm{~d} \mathcal{L}^{n} \leq \int_{B_{R}^{+}}\left|\nabla \partial_{y}^{k-1} v\right|^{2} \mathrm{~d} \mathcal{L}^{n} \\
& \leq \frac{C}{R^{2}} \int_{B_{2 R}^{+}}\left(\left|\nabla \partial_{y}^{k-1} v\right|^{2}+\left|\partial_{y}^{k-1} v\right|^{2}\right) \mathrm{d} \mathcal{L}^{n} \\
& \leq \frac{C}{R^{2}} \int_{B_{2 R}^{+}}\left(\left|\nabla \partial_{y}^{k-1} v\right|^{2}+\left|\nabla \partial_{y}^{k-2} v\right|^{2}\right) \mathrm{d} \mathcal{L}^{n} \\
& \leq \frac{C}{R^{4}} \int_{B_{4 R}^{+}}\left(\left|\nabla \partial_{y}^{k-1} v\right|^{2}+\left|\partial_{y}^{k-1} v\right|^{2}+\left|\nabla \partial_{y}^{k-2} v\right|^{2}+\left|\partial_{y}^{k-2} v\right|^{2}\right) \mathrm{d} \mathcal{L}^{n} \\
& \leq \cdots \leq \frac{C_{\ell, k}}{R^{2 \ell}} \sum_{j=1}^{\ell} \int_{B_{2^{+}}^{+}}\left(\left|\nabla \partial_{y}^{k-j} v\right|^{2}+\left|\partial_{y}^{k-j} v\right|^{2}\right) \mathrm{d} \mathcal{L}^{n} .
\end{aligned}
$$

By choosing $k=\ell$, (A.2) yields

$$
\int_{B_{R}^{+}}\left|\partial_{y}^{k} v\right|^{2} \mathrm{~d} \mathcal{L}^{n} \leq \frac{C_{k}}{R^{2 k}} \mathcal{L}^{n}\left(B_{2^{k} R}^{+}\right) \leq C_{k} R^{n-2 k}
$$

If $k>2 n$, this yields for $R \rightarrow \infty$ : 


$$
\int_{\mathbb{R}_{+}^{n}}\left|\partial_{y}^{k} v\right|^{2} \mathrm{~d} \mathcal{L}^{n}=0
$$

Hence $\partial_{y}^{k} v=0$. This implies

$$
\partial_{y_{i}} \partial_{y}^{k-1} v=0, \text { for } i=1, \ldots, i-1 .
$$

Therefore $\partial_{y}^{k-1} v(y, t)$ is independent of $y \in \mathbb{R}^{n-1}$ and $t \mapsto \partial_{y}^{k-1} v(y, t)$ satisfies the assumptions of Lemma A.1. Hence, we also have

$$
\partial_{y}^{k-1} v=0 .
$$

Especially we have

$$
\partial_{y_{i}} \partial_{y}^{k-2} v=0
$$

for all $i=1, \ldots, n-1$. Hence again $\partial_{y}^{k-2} v(y, t)$ is independent of $y$ and therefore again satisfies the assumptions of Lemma A.1. Iterating this final process yields

$$
v=0 \text {, }
$$

which is the desired conclusion.

Remark: The proof above works, because both $\Delta^{2}$ and $-\Delta$ are monotone operators which are added correctly. If we would destroy this monotonicity by, e.g. examining $\Delta^{2}+\Delta$, Theorem A.1 is not true anymore. For example, a bounded nontrivial solution to $\Delta^{2} v+\Delta v=0$, is $(y, t) \mapsto 1-\cos t$.

Proposition A.3 Let $u: \overline{\mathbb{R}_{+}^{n}} \rightarrow \mathbb{R}$ be a smooth solution of the fourth-order boundary-value problem

$$
\begin{gathered}
\Delta^{2} u-\Delta u=0 \quad \text { in } \mathbb{R}_{+}^{n}, \\
u, \partial_{n} u=0 \quad \text { on } \mathbb{R}^{n-1} \times\{0\} .
\end{gathered}
$$

Furthermore, let $u$ for some $\beta \in \mathbb{R}$ and the corresponding linear function $u_{0}:(y, t) \mapsto \beta t$ satisfy

$$
u-u_{0} \in L^{\infty}\left(\mathbb{R}_{+}^{n}\right)
$$

Then, $u$ is one dimensional, that is

$$
u(y, t)=\beta\left(e^{-t}-1+t\right) \quad \text { for } y \in \mathbb{R}^{n-1}, t \geq 0,
$$

in particular

$$
\Delta u \equiv \beta \quad \text { in } \mathbb{R}^{n-1} \times\{0\}
$$

and

$$
u>0 \quad \text { in } \mathbb{R}_{+}^{n}, \quad \text { if } \beta>0 .
$$

Proof We put 


$$
v(y, t):=u(y, t)-\beta\left(e^{-t}-1+t\right) \quad \text { for } y \in \mathbb{R}^{n-1}, t \geq 0,
$$

and see $v \in C_{l o c}^{\infty}\left(\overline{\mathbb{R}_{+}^{n}}\right)$ and

$$
\begin{gathered}
\Delta^{2} v-\Delta v=0 \quad \text { in } \mathbb{R}_{+}^{n}, \\
v, \partial_{n} v=0 \quad \text { on } \mathbb{R}^{n-1} \times\{0\}, \\
v=u-u_{0}-\beta(\exp (-.)-1) \in L^{\infty}\left(\mathbb{R}_{+}^{n}\right) .
\end{gathered}
$$

Then, the uniqueness in Proposition A.1 gives $v \equiv 0$, which is (A.4), and by direct calculation

$$
\Delta u(y, 0)=\left.\partial_{t t}\left(\beta\left(e^{-t}-1+t\right)\right)\right|_{t=0}=\beta \quad \text { for } y \in \mathbb{R}^{n-1},
$$

which is (A.5). For $\beta>0$, we get by the strict convexity of the exponential function

$$
u(y, t)=\beta\left(e^{-t}-1+t\right)>0 \quad \text { for } y \in \mathbb{R}^{n-1}, t>0,
$$

which is (A.6).

Funding Open Access funding enabled and organized by Projekt DEAL.

Open Access This article is licensed under a Creative Commons Attribution 4.0 International License, which permits use, sharing, adaptation, distribution and reproduction in any medium or format, as long as you give appropriate credit to the original author(s) and the source, provide a link to the Creative Commons licence, and indicate if changes were made. The images or other third party material in this article are included in the article's Creative Commons licence, unless indicated otherwise in a credit line to the material. If material is not included in the article's Creative Commons licence and your intended use is not permitted by statutory regulation or exceeds the permitted use, you will need to obtain permission directly from the copyright holder. To view a copy of this licence, visit http://creativecommons.org/licenses/by/4.0/.

\section{References}

1. Agmon, S., Douglis, A., Nirenberg, L.: Estimates near the boundary for solutions of elliptic partial differential equations satisfying general boundary conditions I. Commun. Pure Appl. Math. 12, 623-727 (1959). https://doi.org/10.1002/cpa.3160120405

2. Agmon, S., Douglis, A., Nirenberg, L.: Estimates near the boundary for solutions of elliptic partial differential equations satisfying general boundary conditions II. Commun. Pure Appl. Math. 17, 35-92 (1964). https://doi.org/10.1002/cpa.3160170104

3. Almansi, E.: Sull'integrazione dell'equazine differenziale $\Delta^{2} \Delta^{2}=0$. Rom. Acc. L. Rend. 81(5), 104-107 (1899)

4. Antunes, P.R., Buoso, D., Freitas, P.: On the behavior of clamped plates under large compression. SIAM J. Appl. Math. 79, 1872-1891 (2019). https://doi.org/10.1137/19M1249606

5. Bickley, W.G.: Deflexions and vibrations of a circular elastic plate under tension. Philos. Mag. 7(15), 776797 (1933). https://doi.org/10.1080/14786443309462222

6. Boggio, T.: Sulle funzioni di Green d'ordine, m. Rend. Circ. Mat. Palermo 20, 97-135 (1905). https://doi.org/ 10.1007/BF03014033

7. Bucur, D., Gazzola, F.: The first Biharmonic Steklov eigenvalue: positivity preserving and shape optimization. Milan J. Math. 79, 247-258 (2011). https://doi.org/10.1007/s00032-011-0143-X

8. Buoso, D., Kennedy, J.: The Bilaplacian with Robin boundary conditions (2021). arXiv:2105.11249

9. Cassani, D., Tarsia, A.: Maximum principle for higher order operators in general domains (2020). arXiv: 2011.01091v1 
10. Dall'Acqua, A., Sweers, G.: On domains for which the clamped plate system is positivity preserving. Differential Equations and Inverse Problems, ed. by Carlos Conca, Raul Manasevich, Gunter Uhlmann and Michael Vogelius, AMS (2004). https://doi.org/10.1090/conm/362/06609

11. Douglis, A., Nirenberg, L.: Interior estimates for elliptic systems of partial differential equations. Commun. Pure Appl. Math. VIII, pp. 503-538 (1955). https://doi.org/10.1002/cpa.3160080406

12. Duffin, R.J.: On a question of Hadamard concerning super-biharmonic functions. J. Math. Phys. 27, 253258 (1948). https://doi.org/10.1002/sapm1948271253

13. Frank, L.S.: Coercive singular perturbations I: a-priori estimates. Ann. Mat. Pura Appl. IV 119, 41-113 (1979). https://doi.org/10.1007/BF02413170

14. Garabedian, P.R.: A partial differential equation arising in conformal mapping. Pac. J. Math, 1, pp 485524 (1951)

15. Gazzola, F., Grunau, H.-Ch., Sweers, G.: Problems, polyharmonic boundary value.: Springer. Lecture Notes in Mathematics 1991 (2010). https://doi.org/10.1007/978-3-642-12245-3

16. Gilbarg, D., Trudinger, N.S.: Equations, elliptic partial differential, of second order: Springer, 3. Edition, Berlin, Heidelberg, New York, Tokyo (1998). https://doi.org/10.1007/978-3-642-61798-0

17. Greenlee, W.: Rate of convergence in singular perturbations. Ann. de l'Institut Fourier Tome 18, 135-191 (1968). https://doi.org/10.5802/aif.296

18. Grunau, H.-Ch.: Optimal estimates from below for green functions of higher order elliptic operators with variable leading coefficients. Arch. Math. (2021). https://doi.org/10.1007/s00013-021-01597-x

19. Grunau, H.-Ch.: Positivity, change of sign and buckling eigenvalues in a one-dimensional fourth order model problem. Adv. Differ. Equ. 7, 177-196 (2002)

20. Grunau, H.-Ch., Robert, F.: Positivity and almost positivity of biharmonic Green's functions under Dirichlet boundary conditions. Arch. Ration. Mech. Anal. 195, 865-898 (2010). https://doi.org/10.1007/ s00205-009-0230-0

21. Grunau, H.-Ch., Robert, F., Sweers, G.: Optimal estimates from below for biharmonic Green functions. Proc. Am. Math. Soc. 139, 2151-2161 (2011). https://doi.org/10.1090/S0002-9939-2010-10740-2

22. Grunau, H.-Ch., Romani, G., Sweers, G.: Differences between fundamental solutions of general higher order elliptic operators and of products of second order operators. Math. Ann. (2020). https://doi.org/10. 1007/s00208-020-02015-3

23. Grunau, H.-Ch., Sweers, G.: A clamped plate with a uniform weight may change sign. Discrete Contin. Dyn. Syst. S 7(4), 761-766 (2014). https://doi.org/10.3934/dcdss.2014.7.761

24. Grunau, H.-Ch., Sweers, G.: In any dimension a "clamped plate" with a uniform weight may change sign. Nonlinear Anal. A: T.M.A, 97, pp. 119-124 (2014). https://doi.org/10.1016/j.na.2013.11.017

25. Gueugnon, M.: Perturbations singulieres dans les espaces $L^{p}$ pour l'opérateur $\varepsilon \Delta^{2}-\Delta+I$. C.R. Acad. Sci. Paris 293, pp. 129-131 (1981)

26. Hadamard, J.: Mémoire sur le problème d'analyse relatif à l'équibilibre des plaques élastiques encastrées, Euvres de Jaques Hadamard, Tome II, CNRS Paris, (1968), pp. 515-641, Reprint of: Mémoire présentés par divers savant a l'Académie des Sciences (2), (1908), 33:1-128

27. Hadamard, J.: Sur certains cas intéressants du problème biharmonique, Euvres de Jaques Hadamard, Tome III, CNRS Paris: pp. 1297-1299, Reprint of: Atti IV Congr. Intern. Mat. Rome 1908, 12-14 (1968)

28. Himmel, B.: Die Boggio-Formel für polyharmonische Dirichlet-Probleme, Bachelor thesis: Advisor H.-Ch. Grunau, Otto-von-Guericke Universität Magdeburg (2019). http://www-ian.math.uni-magdeburg. de/home/grunau/papers/Himmel_Bachelor.pdf

29. Najman, B.: Singular perturbations of elliptic boundary value problems in $L^{p}$. Glas. Mat. Ser. III 23(43), 259-290 (1988)

30. Pulst, L.: Dominance of positivity of the Green's function associated to a perturbed polyharmonic Dirichlet boundary value problem by pointwise estimates, $\mathrm{PhD}$ thesis: advisor H.-Ch. Grunau, Otto-vonGuericke Universität Magdeburg (2015). https://doi.org/10.25673/4208

Publisher's Note Springer Nature remains neutral with regard to jurisdictional claims in published maps and institutional affiliations. 